Authors

Dirk Müller, Eric A. Cornell, Marco Prevedelli, Peter D. D. Schwindt, Ying-Ju Wang, and Dana Z. Anderson 


\title{
Magnetic switch for integrated atom optics
}

\author{
Dirk Müller, Eric A. Cornell,* Marco Prevedelli, ${ }^{\dagger}$ Peter D. D. Schwindt, Ying-Ju Wang, and Dana Z. Anderson \\ Department of Physics and JILA, University of Colorado, Boulder, Colorado 80309-0440 \\ and National Institute of Standards and Technology, Boulder, Colorado 80309
}

(Received 6 November 2000; published 15 March 2001)

\begin{abstract}
A magnetic waveguide structure allows switching of neutral atoms between two guides. The switch consists of lithographically patterned current-carrying wires on a sapphire substrate. By selectively sending current through a particular set of wires, we select the desired output port of an incoming beam. We utilize two different magnetic-guiding schemes to adiabatically manipulate the atom trajectory.
\end{abstract}

DOI: 10.1103/PhysRevA.63.041602

PACS number(s): 03.75.Be, 32.80.Pj

Recent progress in laser cooling has stimulated advances in neutral-atom optics. By using either photon-atom or magnetic-field interactions, beam splitters [1], mirrors [2-4], lenses [5,6], and waveguides [7-12] have been demonstrated. Integrated atom optics aims to miniaturize atom optics devices into a modular and compact form, similar to the way integrated optics has miniaturized optical setups. By using photolithographically patterned current-carrying wires, magnetic guides $[13,14]$, beam splitters $[15,16]$, and even an atom motor [17] have been demonstrated. Well characterized atom waveguides and beam splitters may make possible inertial and rotation measurements of exquisite sensitivity via large-enclosed-area atom interferometers [18]. Further, a combination of waveguides and switches could be used to implement a possible neutral-atom storage ring. Such a storage ring could be used to create a monochromatic beam of neutral atoms, analogous to storage rings for charged particles.

Here we report on a switch for neutral atoms that is an extension of our previously reported waveguide for neutral atoms [13]. A beam of laser-cooled atoms is electromagnetically guided along a one-dimensional potential minimum. The switch directs the magnetic-field minimum toward either one of two output ports, selected by sending current along one or another auxiliary wire.

The input and output guides have the same structure. This configuration allows for expansion to other atom-optics devices before and after the switch region. The switch itself utilizes two different guiding schemes, referred to as the center guide [Fig. 1(a)] and the side guide [Fig. 1(b)]. The center guide operates as described in Ref. [13]. It offers the strongest confinement and potential-field gradient for a given geometry and wire current. The side guide suffers from a smaller field gradient compared to the center guide, but allows easy transfer of atoms from one potential minimum into another. The combination of the center guide and the side guide allows us to switch an atom beam between two ports with a 8-mm output separation.

\footnotetext{
*Also at Quantum Physics Division, National Institute of Standards and Technology, Boulder, CO 80309.

†Permanent address: Dipartimento di Fisica, Dell' Università di Firenze, Largo Enrico Fermi 2, 50125 Firenze, Italy.
}

Initially, weak-field-seeking atoms are guided by a center guide. The center guide consists of two $100 \times 100-\mu \mathrm{m}$ wires spaced 200 microns from center to center with currents running in the same direction [Fig. 1(a)]. The resulting magnetic field is zero at the center between the wires and increases linearly outward. A small longitudinal field is applied to prevent the field magnitude from vanishing at the track center. The maximum transverse guiding potential increases linearly with applied current. The transverse magnetic-field gradient around the center is proportional to the track current and inversely proportional to the square of the wire spacing.

In the switch region atoms are guided by a side guide. The side guide consists again of two $100 \times 100-\mu \mathrm{m}$ wires spaced $200 \mu \mathrm{m}$ from center to center, but, in contrast to the center guide, with currents running in opposing directions. The 3-4 times larger current of the auxiliary wire creates a bias field for the primary wire. This bias field creates a magnetic field minimum on the side of the primary wire facing away from the auxiliary wire [Fig. 1(b)]. To achieve a trap depth and potential gradient equivalent to the center guide, larger currents are required in the side-guide configuration. This feature makes the side guide less desirable for transporting atoms from one region on the substrate to another or around a

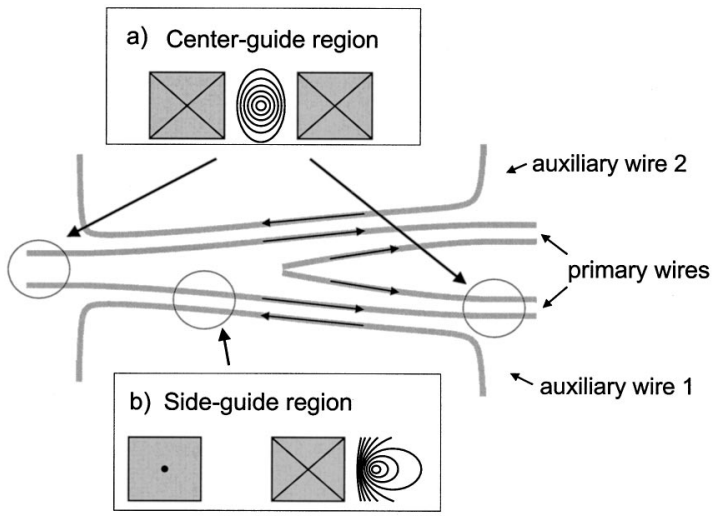

FIG. 1. Schematic of switch (not to scale). Insets (a) and (b) show the magnetic-field contour lines for each region at a primarywire current of $3.11 \mathrm{~A}$ and an auxiliary-wire current of $14.0 \mathrm{~A}$. The contour lines are spaced by $10 \mathrm{G}$. The switch starts and ends with a center guide (a). In the middle of the switch, atoms are transferred from a center guide to a side guide (b). Depending on which one of the two auxiliary wires 1 and 2 is turned on, the atoms exit port 1 or 2 , respectively. The two output ports are separated by $8 \mathrm{~mm}$. 


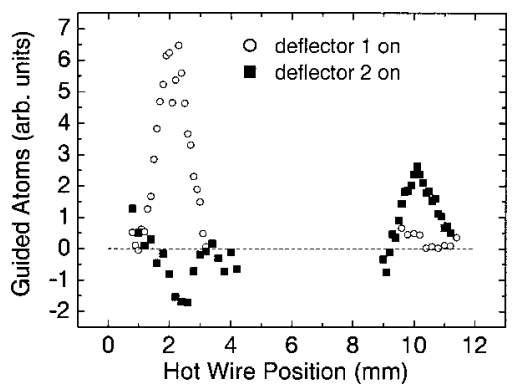

FIG. 2. Switch output. The open circles show the observed flux with auxiliary wire 1 on and auxiliary wire 2 off. The solid squares show the flux with auxiliary wire 1 off and auxiliary wire 2 on. In each case the disabled port shows very little background flux. Negative background is due to a background subtraction. The output flux differs by a factor of $2-3$ between the two ports.

sharp curve, and hence, we utilize the side guide only in the immediate switch region.

The guiding potentials are switched adiabatically from a center guide to a side guide and back to a center guide as the atoms traverse the switch. After being guided by a center guide for one centimeter, the two center-guide wires start to separate slowly (Fig. 1). This increased wire separation causes a field-gradient decrease in the guiding region. As the center-guide wires (now referred to as the primary wires) separate from each other, they each form one path of the switch. In this region an auxiliary wire approaches the primary wire such that the resulting magnetic field of the switch-port potential minimum remains $100 \mu \mathrm{m}$ from the primary-wire center. Atoms are guided over the next $4 \mathrm{~cm}$ with the side guide until the potential starts to merge back into a center guide over an additional 4-cm length, reversing the above process. While the atoms follow the side guide, they are bent away from the symmetry axis of the switch to achieve an 8-mm separation between the two output ports of the switch. After the switch region, atoms are transferred back into a center-guide configuration for the remaining $1-\mathrm{cm}$ guiding distance toward the output port.

A modified magneto-optical trap (MOT) serves as our source of laser-cooled atoms [19] for the switch experiment. A diode laser in a master-oscillator power-amplifier configuration (MOPA) [20] provides $350 \mathrm{~mW}$ of single-frequency light tuned near the $5 S_{1 / 2}(F=2) \rightarrow 5 P_{3 / 2}\left(F^{\prime}=3\right)$ transition in rubidium for trapping and cooling in the MOT. This light is divided into three beams, which are directed into the chamber along orthogonal axes, and retroreflected to supply cooling along all directions. A 30-mW external-cavity diode laser [21] supplies light tuned to the $5 S_{1 / 2}(F=1)$ $\rightarrow 5 P_{3 / 2}\left(F^{\prime}=2\right)$ transition to repump atoms that fall into the $F=1$ ground state back into the cycling transition. A 500$\mu \mathrm{m}$ hole is drilled in the center of one of the retroreflecting mirrors, and this mirror is placed inside the vacuum chamber. Thus, one of the six confining laser beams has a dark region in the center of its cross section. The radiationpressure imbalance for atoms in the MOT that enter into the shadow of the hole accelerates those atoms toward and then through the hole in the mirror. The resulting atomic beam is referred to as a low-velocity intense source (LVIS) [22]. Our

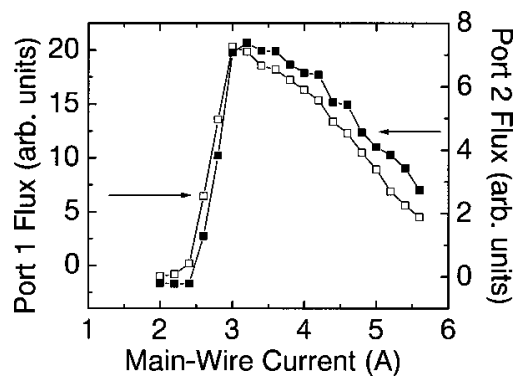

FIG. 3. Guided atoms versus primary-wire current. At a constant auxiliary-wire current, we vary the primary-wire current and measure guided atoms for each port. The flux peaks at a current ratio of 4.4 and 4.7 for port 1 (open squares) and 2 (solid squares), respectively.

observations show that $90 \%$ of the LVIS flux atoms are optically pumped into the $F=1$ ground state by the MOT light. We observe that roughly $50 \%$ are in the $m_{F}=0$ state and the rest of the atoms are roughly equally divided between the two $m_{F}= \pm 1$ sublevels. Therefore, only $25 \%$ of LVIS atoms are in the correct state to be guided. We estimate the transverse-velocity distribution entering our guide to be about $v_{t}=5.0 \pm 2.0 \mathrm{~cm} / \mathrm{s}$ [22]. A time-of-flight measurement found the longitudinal velocity of LVIS to be $v_{l}=10.1$ $\pm 2.0 \mathrm{~m} / \mathrm{s}$.

We characterize the performance of our switch by measuring the atom flux from both output ports with the primary wires and one of the auxiliary wires turned on. For the flux measurement, atoms coming out of the guide are ionized by the hot wire and the subsequent ions are then detected by a channeltron. The $70-\mu \mathrm{m}$-diameter hot wire placed $\sim 2.5 \mathrm{~cm}$ from the output of the switch intercepts a small fraction of the diverging atomic beam. To resolve the output profile of our switch, we move the hot wire transverse to the output beams. With $4.5 \mathrm{~A}$ in the primary wires and 14.0 $\mathrm{A}$ in auxiliary wire 1 , we measure the flux out of both output ports (Fig. 2). To avoid overheating of the wires, we conduct our experiment in a pulsed mode with $45 \mathrm{~ms}$ current pulses at a 1 -s repetition rate. We observe from port 1 a total flux of $6.3 \times 10^{4}$ atoms/s and only a small background flux from port 2. Under the same conditions, we turn off auxiliary wire 1 , and instead, send 14.0 A through auxiliary wire 2. We observe a total flux of $3.1 \times 10^{4}$ atoms/s from port 2 and a negative background flux out of port 1 . The negative flux is due to our background subtraction process. The data show that we can direct atoms to either port by turning on the appropriate auxiliary wire. We attribute the factor of 2-3 difference in total flux between the two ports to the initial pointing of the LVIS atoms and possible contamination between the wires blocking atoms.

To characterize the guiding properties of our switch, we hold the auxiliary-wire current constant at 14.0 A and observe the flux from each port while varying the primary-wire current. For a specific auxiliary-wire current, there is an optimum primary-wire current that maximizes the guided-atom flux (Fig. 3). For very large primary-wire currents, the magnetic-field minimum is shifted far away from the primary wire, resulting in a reduced field gradient. When the 
primary-wire current is too low, the generated magnetic-field gradient is sufficient to bend the atoms around the curve, but the field minimum is close to the wire surface and atomsurface interactions result in a lower flux. Our data show an optimum ratio between auxiliary-wire and primary-wire current of 4.7 for port 1 and 4.4 for port 2. The agreement between the two output ports suggests that the flux difference is not due to an asymmetry in our switch design, but, rather, due to dirt contamination in port 2.

In conclusion, we demonstrated a switch for integrated atom optics that can direct a neutral atom beam to either of two output ports. The switch is created by using photolitho- graphically patterned current-carrying wires, which makes it easily reproducible. We transfer atoms from a center-guide configuration to a side guide and back again to a center guide. There is qualitative agreement between the guiding concept and our observed data.

The authors thank Carl Wieman for helpful discussions. This work was made possible by funding from the Office of Naval Research (Grant No. N00014-94-1-0375), the National Science Foundation (Grant No. Phy-95-12150), and a Multi University Research Initiative through the Department of Defense (MURI Grant No. DAAD 19-00-0163).
[1] M. Kasevich and S. Chu, Phys. Rev. Lett. 67, 181 (1991).

[2] W. Seifert, C. S. Adams, V. I. Balykin, C. Heine, Y. Ovchinnikov, and J. Mlynek, Phys. Rev. A 49, 3814 (1994).

[3] T. M. Roach, H. Abele, M. G. Boshier, H. L. Grossman, K. P. Zetie, and E. A. Hinds, Phys. Rev. Lett. 75, 629 (1995).

[4] C. V. Saba, P. A. Barton, M. G. Boshier, I. G. Hughes, P. Rosenbusch, B. E. Sauer, and E. A. Hinds, Phys. Rev. Lett. 82, 468 (1999).

[5] G. Timp, R. E. Behringer, D. M. Tennant, J. E. Cunningham, M. Prentiss, and K. K. Berggren, Phys. Rev. Lett. 69, 1636 (1992).

[6] W. G. Kaenders, F. Lison, I. Muller, A. Richter, R. Wynands, and D. Meschede, Phys. Rev. A 54, 5067 (1996).

[7] M. J. Renn, D. Montgomery, O. Vdovin, D. Z. Anderson, C. E. Wieman, and E. A. Cornell, Phys. Rev. Lett. 75, 3253 (1995).

[8] M. J. Renn, E. A. Donley, E. A. Cornell, C. E. Wieman, and D. Z. Anderson, Phys. Rev. A 53, R648 (1996).

[9] J. Schmiedmayer, Phys. Rev. A 52, R13 (1995).

[10] J. Denschlag, D. Cassettari, and J. Schmiedmayer, Phys. Rev. Lett. 82, 2014 (1999).

[11] J. Denschlag, D. Cassettari, A. Chenet, S. Schneider, and J. Schmiedmayer, Appl. Phys. B: Lasers Opt. 69, 291 (1999).

[12] M. Key, I. G. Hughes, W. Rooijakkers, B. E. Sauer, E. A. Hinds, D. J. Richardson, and P. G. Kazansky, Phys. Rev. Lett.
84, 1371 (2000).

[13] D. Müller, D. Z. Anderson, R. J. Grow, P. D. D. Schwindt, and E. A. Cornell, Phys. Rev. Lett. 83, 5194 (1999).

[14] N. H. Dekker, C. S. Lee, V. Lorent, J. H. Thywissen, S. P. Smith, M. Drndic, R. M. Westervelt, and M. Prentiss, Phys. Rev. Lett. 84, 1124 (2000).

[15] D. Müller, E. A. Cornell, M. Prevedelli, P. D. D. Schwindt, A. Zozulya, and D. Z. Anderson, Opt. Lett. 25, 1382 (2000).

[16] D. Cassettari, B. Hessmo, R. Folman, T. Maier, and J. Schmiedmayer, Phys. Rev. Lett. 85, 5483 (2000).

[17] J. Reichel, W. Hänsel, P. Hommelhoff, and T. W. Hänsch, e-print quant-ph/0008118.

[18] J. Schmiedmayer et al., Atom Interferometry, edited by P. R. Berman (Academic, Boston, 1997).

[19] E. L. Raab, M. Prentiss, A. Cable, S. Chu, and D. E. Pritchard, Phys. Rev. Lett. 59, 2631 (1987); C. Monroe, W. Swann, H. Robinson, and C. E. Wieman, ibid. 65, 1571 (1990).

[20] D. Mehuys, D. F. Welch, and L. Goldberg, Electron. Lett. 28, 1944 (1992), and J. N. Walpole, Opt. Quantum Electron. 28, 623 (1996).

[21] K. B. MacAdam, A. Steinbach, and C. Wieman, Am. J. Phys. 60, 1098 (1992).

[22] Z. T. Lu, K. L. Corwin, M. J. Renn, M. H. Anderson, E. A. Cornell, and C. E. Wieman, Phys. Rev. Lett. 77, 3331 (1996). 